\title{
Effect of Frank-Kamenetskii Parameter on the Flames with Chain- Breaking and Chain-Branching Kinetics
}

\footnotetext{
${ }^{1}$ Waheed A. A.

${ }^{2}$ Akinpelu F. O.
}

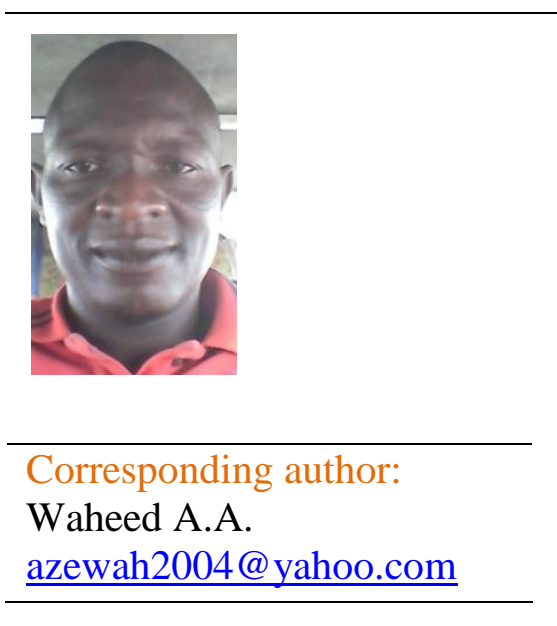

Received: June 07, 2017

Revised: June 29, 2017

Published: June 30, 2017

\begin{abstract}
This paper presents the effects of Frank- Kamenetskii parameter on Flames with Chain- breaking and Chain-branching kinetics. The combustion equation describing the phenomenon was nondimensionnalised to arrive at dimensionless equations. The existence and uniqueness of the solution was proved using upper and lower solution method .The properties of equation were also examined. The numerical solution showed that the steady problem has at least two solutions under certain conditions using finite difference scheme and Runge- kutta of order four and the result obtained were presented graphically. It was observed that temperature profile increases as the FrankKamenetskii increases.
\end{abstract}

Keywords- Combustion, Flames, Chain-breaking, Chain branching. 


\section{INTRODUCTION}

Combustion waves have been studied for several years and still a subject or research. They have been observed in numerous experiments and play an important role in industrial processes, such as one of the current technologies for creating advanced materials", Self-propagating Hightemperature Synthesis (SHS)

Jang T. and Raduleson M.I. (2012) investigated dynamics of shock induced ignition in Fickett's model with chainbranching kinetics. A close form analytical solution was obtained by the method of characteristics and high activation energy asymptotic.

Huangwei and Zheng (2011) investigated spherical flame initiation and propagation with thermally sensisitive intermediate kinetics. The analytical result and simulation of result were obtained. Flame spherical propagation showed to be strongly affected by the Lewis number of fuel and radicals as well as the heat of reaction.

Ayeni and Waheed (2005) examined a mathematical model of cigarette-like combustion using high activation energy asymptotics .Of particular interest were question of existence and uniquiness.It showed that the steady temperature increases as the Frank-Kamenetskii parameter $\lambda_{1}$ increases.

Olayiwola ,Olatunji,Ajao,Waheed and Lanlege (2013) investigated effect of FrankKamenetskii parameter on the propagation of forward and opposed shouldering combustion .The properties of solution was examined under certain condition while the equation was solved analytically using asymptotic expansion. It was discovered that Frank-Kamenetskii parameter played a crucial role in the slow burning process and the temperature decreased and species is consumed in the spatial direction.

Olarenwaju, P. O., Ayeni, R. O., Adesanya A. O., Fenugi, O. J. and Adegbile, E. A (2007) examined the effect of activation emerges and strong viscous dissipation term on two-step Arrhenius combustion reactions to give further insight into the theory of combustion under physical reasonable assumption. They extend the non-uniformly of vessels discussed in Olarewaju (2007). In a uniform vessel, maximum temperature occurs towards the end of the tube on the other hand, in a uniform vessel, maximum temperature occurs at the centre. Also maximum temperature for diverging or converging channel is greater than that of a uniform vessel.

Olarewaju P.O. (2005) examined solutions of two-step reactions with variable thermal conductivity. He considered not only the generalized temperature dependences of reaction rate, but he also proposed suitable approximation of the kinetics reactions in the limit of large/small activation energy. Gubernov, V. V. and Kim, J. S. (2006) studied the steady travelling waves in the adiabatic model with two-step chain branching reaction mechanisms was investigated numerically. The properties of these solutions were demonstrated to have similarities with the properties of nonadiabatic combustion waves. That is, there is a residual amount of fuel left behind the travelling waves and the solutions can exhibit extinction. It is also shown that the model processes a new type multiple travelling wave solutions( which one call wave trains) with complex structured of the profiles and very speeds 


\section{MATHEMATICAL FORMULATION}

The mathematical equations describing the flames with Chain-breaking and Chain-branching Kinetics is given by;

$$
\rho c_{p} \frac{\partial T}{\partial t}=K \frac{\partial^{2} T}{\partial x^{2}}+\frac{\left(T-T_{0}\right)^{n} Q A e^{\frac{-E}{R T}}}{a+b e^{\frac{-E}{R T}}}
$$

with initial and boundary conditions,

$\mathrm{T}(\mathrm{x}, 0)=\mathrm{T}_{0} \quad \mathrm{~T}(0, \mathrm{t})=\mathrm{T}_{0}, \mathrm{~T}(\mathrm{~L}, \mathrm{t})=\mathrm{T}_{0}$

\section{METHOD OF SOLUTION}

We make the variable dimensionless by introducing

$\theta=\frac{E}{R T_{O}^{2}}\left(T-T_{0}\right), \quad x^{1}=\frac{x}{L} \quad$ and $\quad \varepsilon=\frac{R T_{0}}{E}$,

and we assume that,

$\mathrm{E}_{1}=E+\varepsilon E$

$\theta=\frac{1}{\varepsilon T_{0}}\left(T-T_{0}\right)$

The equation becomes

$\frac{\partial \theta}{\partial t}=K \frac{\partial^{2} \theta}{\partial x^{2}}+\frac{\lambda_{1} \theta^{n} e^{\frac{\theta}{1+\varepsilon \theta}}}{1+\lambda_{2} e^{\frac{\theta-1}{1+\varepsilon \theta}}}$

with initial and boundary conditions

$\theta(x, 0)=0, \theta(0, t)=0, \theta(1, t)=0$

where,

$K=\frac{k}{\rho c_{p} L^{2}} \quad$ is the scaled thermal Conductivity

$\lambda_{1}=\frac{\left(\varepsilon T_{0}\right)^{n} A Q e^{\frac{-E}{R T_{0}}}}{\rho c_{p} \varepsilon T_{0} a}$ is the Frank - Kamenentskii parameter 


$$
\lambda_{2}=\frac{b}{a} e^{\frac{-E_{1}}{R T_{0}}} \text { is the dimensionless permeability parameter }
$$

\section{Existence and Uniqueness of Solution.}

Definition 1: A solution function $\mathrm{v}$ is said to be a lower solution of the problem $\mathrm{LV}=\mathrm{F} \quad(\mathrm{y}, \mathrm{t}, \mathrm{v})$

where,

$\mathrm{L} \equiv \frac{\partial}{\partial t}-\left(a,(y, t) \frac{\partial}{\partial t}+b(y, t) \frac{\partial^{2}}{\partial y^{2}}+c(y, t)\right.$

If $\mathrm{v}$ satisfies $\mathrm{LV} \leq F(y, t, v)$

$v(y, 0) \leq F(y), v(0, t) \leq h(y), v(\infty, t) \leq h(y)$

Definition 2: A smooth function $U$ is said to be an upper solution of the problem

$\mathrm{LU}=\mathrm{F} \quad(\mathrm{y}, \mathrm{t}, \mathrm{u})$

Where

$\mathrm{L} \equiv \frac{\partial}{\partial t}-\left(a,(y, t) \frac{\partial}{\partial t}+b(y, t) \frac{\partial^{2}}{\partial y^{2}}+c(y, t)\right.$

if $\mathrm{u}$ satisfies

$\mathrm{LU} \geq F(y, t, u)$

$u(y, 0) \geq F(y), u(0, t) \geq h(y), u(\infty, t) \geq h(y)$

\section{Theorem 3.1.1}

Let $\mathrm{k}>0, \lambda_{1}>0, \lambda_{2}>0, \in>0, n=0$. Then Equation (3.4) with the boundary and initial condition has a solution for all $\mathrm{t} \geq 0$.

Proof:

Let

$$
L \theta=f(x, t, \theta)
$$

where

$$
L \theta=\frac{\partial \theta}{\partial t}-K \frac{\partial^{2} \theta}{\partial x^{2}}
$$

$$
f(x, t, \theta)=\frac{\lambda_{1} e^{\frac{\theta}{1+\varepsilon \theta}}}{1+\lambda_{2} e^{\frac{\theta-1}{1+\varepsilon \theta}}}
$$

$$
\underline{\theta}(\mathrm{x}, \mathrm{t})=0
$$


we shall show that $\underline{\theta}(\mathrm{x}, \mathrm{t})=0$ is a lower solution.

Clearly,

$\underline{\theta}(\mathrm{x}, \mathrm{t})=0, \underline{\theta}(\mathrm{x}, \mathrm{t})=\underline{\theta}(1, \mathrm{t})$

Now,

$$
\frac{\partial \theta}{\partial t}=\frac{\partial^{2} \theta}{\partial x^{2}}=0
$$

This implies

$$
\begin{aligned}
L \theta & =\frac{\partial \theta}{\partial t}-K \frac{\partial^{2} \theta}{\partial x^{2}}=0-0=0 \\
f(x, t, \theta) & =\frac{\lambda_{1}}{1+\lambda_{2} e^{-1}}
\end{aligned}
$$

Hence,

$$
L \theta \leq f(x, t, \theta)
$$

By definition $1, \underline{\theta}(\mathrm{x}, \mathrm{t})=0$ is a lower solution

Also consider

$$
\bar{\theta}(\mathrm{x}, \mathrm{t})=\left(\frac{\lambda_{1} e^{\frac{1}{\varepsilon}}}{1+\lambda_{2} e^{\frac{1}{\varepsilon}}}\right) t
$$

We shall show that $\bar{\theta}(\mathrm{x}, \mathrm{t})$ as defined is an upper solution Clearly,

$$
\bar{\theta}(\mathrm{x}, 0)=0, \bar{\theta}(0, \mathrm{t})=\frac{\lambda_{1} e^{\frac{1}{\varepsilon}} t}{1+\lambda_{2} e^{\frac{1}{\varepsilon}}}, \bar{\theta}(1, \mathrm{t})=\frac{\lambda_{1} e^{\frac{1}{\varepsilon}} t}{1+\lambda_{2} e^{\frac{1}{\varepsilon}}}
$$

Now,

$$
\begin{gathered}
\frac{\partial \theta}{\partial t}=\frac{\lambda_{1} e^{\frac{1}{\varepsilon}}}{1+\lambda_{2} e^{\frac{1}{\varepsilon}}} \\
\frac{\partial^{2} \theta}{\partial t^{2}}=0
\end{gathered}
$$

This implies

$$
\mathrm{L} \bar{\theta}=\frac{\partial \bar{\theta}}{\partial t}-\mathrm{k} \frac{\partial^{2} \bar{\theta}}{\partial x^{2}}=1+\lambda_{2} e^{\frac{1}{\varepsilon}}
$$




$$
\mathrm{f}(\mathrm{x}, \mathrm{t}, \bar{\theta})=\frac{\lambda_{1} e^{\frac{\theta}{1+\varepsilon \theta}}}{1+\lambda_{2} e^{\frac{\theta-1}{1+\varepsilon \theta}}} \leq \frac{\lambda_{1} e^{\frac{1}{\varepsilon}}}{1+\lambda_{2} e^{\frac{1}{\varepsilon}}}
$$

Hence

By definition 2, $\bar{\theta}(\mathrm{x}, \mathrm{t})=\frac{\lambda_{1} e^{\frac{1}{\varepsilon}}}{1+\lambda_{2} e^{\frac{1}{\varepsilon}}}$ is an upper solution

$\mathrm{L} \bar{\theta} \geq(\mathrm{x}, \mathrm{t}, \bar{\theta})$

Hence, there exists a solution of problem (3.4). This completes the proof.

\section{Theorem 3.1.2}

Let $\varepsilon>0$ and $n=0$.

$$
\frac{\partial \theta}{\partial t}=K \frac{\partial^{2} \theta}{\partial x^{2}}+\frac{\lambda_{1} \theta^{n} e^{\frac{\theta}{1+\varepsilon \theta}}}{1+\lambda_{2} e^{\frac{\theta-1}{1+\varepsilon \theta}}}
$$

then

$$
\frac{d \theta}{d t} \geq 0
$$

we have

$$
\begin{aligned}
& \frac{\partial \theta}{\partial t}=K \frac{\partial^{2} \theta}{\partial x^{2}}+\frac{\lambda e^{\frac{\theta}{1+\varepsilon \theta}}}{1+\lambda_{2} e^{\frac{\theta-1}{1+\varepsilon \theta}}} \\
& \theta(0, t)=\theta(1, t)=0=\theta(x, 0)
\end{aligned}
$$

Then $\frac{\partial \theta}{\partial t} \geq 0$

In the proof, we shall make use of the following lemma of Kolodner and Pederson (1966)

Lemma (Kolodner and Pederson 1966).

Let $u(x, t)=0\left(e^{a / \times\left.\right|^{2}}\right)$ be a solution on $R^{n} \times\left[0, t_{0}\right)$ of differential inequality

$$
\frac{\partial u}{\partial t}-\Delta u+k(x, t) u \geq 0
$$

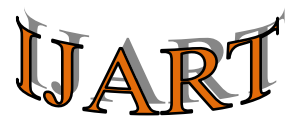


where $\mathrm{k}$ is bounded from below. If $u(x, 0) \geq 0$ then $u(x, t) \geq 0$ for all

\section{Proof:}

Let $n=0$, the Equation (6) becomes

$$
\frac{\partial \theta}{\partial t}=K \frac{\partial^{2} \theta}{\partial x^{2}}+\frac{\lambda_{1} e^{\frac{\theta}{1+\varepsilon \theta}}}{1+\lambda_{2} e^{\frac{\theta-1}{1+\varepsilon \theta}}}
$$

Differentiating with respect to $t$, we have

$$
\begin{aligned}
& \frac{\partial^{2} \theta}{\partial t^{2}}=\frac{\partial}{\partial t}\left(k \frac{\partial^{2} \theta}{\partial x^{2}}\right)+\frac{\partial}{\partial t}\left[\frac{\lambda_{1} e^{\frac{\theta}{1+\varepsilon \theta}}}{1+\lambda_{2} e^{\frac{\theta-1}{1+\varepsilon \theta}}}\right] \\
& \frac{\partial^{2} \theta}{\partial t^{2}}=k \frac{\partial^{2} \theta}{\partial x^{2}}\left(\frac{\partial \theta}{\partial t}\right)+\frac{\partial}{\partial t}\left[\frac{\lambda_{1} e^{\frac{\theta}{1+\varepsilon \theta}}}{1+\lambda_{2} e^{\frac{\theta-1}{1+\varepsilon \theta}}}\right] \frac{\partial \theta}{\partial t}
\end{aligned}
$$

Let $U=\frac{\partial \theta}{\partial t}$ then, can be written as

$$
\frac{\partial U}{\partial t}=k \frac{\partial^{2} U}{\partial x^{2}}-V(x, t) U \geq 0
$$

where $V(x, t)=-\frac{\lambda_{1} \frac{e^{\frac{\theta}{1+\varepsilon \theta}}}{(1+\varepsilon \theta)^{2}}}{\left(1+\lambda_{2} e^{\frac{\theta-1}{1+\varepsilon \theta}}\right)^{2}}$

Clearly V is bounded from below. Hence by Kolodner and Pederson's lemma

$$
U(x, t) \geq 0 \quad \text { i.e } \frac{\partial \theta}{\partial t} \geq 0
$$

This completes the proof.

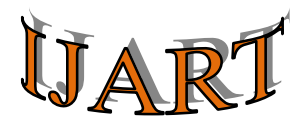


By Theorem 3.1.2 the problem has a solution and the solution is unique.

Theorem 3.1.3: Let $n=\varepsilon=\lambda_{2}=0$

Then, the steady equation

$$
K \frac{\partial^{2} \theta}{\partial x^{2}}+\frac{\lambda e^{\frac{\theta}{1+\varepsilon \theta}}}{1+\lambda_{2} e^{\frac{\theta-1}{1+\varepsilon \theta}}}=0
$$

Which satisfies

$$
\theta(0)=\theta(0)=0
$$

has at least two solutions

\section{Proof:}

Let $n=\varepsilon=\lambda_{2}=0$ in Equation (3.4), and then we have

$$
\begin{gathered}
\frac{d^{2} \theta}{d x^{2}}+\lambda_{1} e^{\theta}=0 \\
\theta(0)=\theta(1)=0,
\end{gathered}
$$

Where

$$
\delta=\frac{\lambda_{1}}{K_{1}}
$$

Then from Buckmaster and Ludford (1982)

$$
\begin{aligned}
& \theta(x)=2 \operatorname{In}\left[e^{1 / 2 \theta m} \operatorname{sechcx}\right] \\
& \theta(0)=2 \operatorname{In}\left[\exp \left(\frac{1}{2} \theta i(0)\right) \sec h c x\right]_{, \mathrm{i}=1},
\end{aligned}
$$

\section{NUMERICAL SIMULATION}

In this section, the sketch of how to obtain the solution on effects of flames with chain -breaking and chain- breaking kinetics was given. The description of the numerical scheme employed in solving the problems (steady case) is shooting method and Runge - kutta of order four. 


\section{Description of the shooting method}

Consider the differential equation

$$
\begin{gathered}
K \theta^{11}(x)+\frac{\lambda_{1(\theta(x))^{n} e \frac{\theta(x)}{1+\varepsilon \theta(x)}}}{1+\lambda_{2} e \frac{\theta(x)-1}{1+\varepsilon \theta(x)}}=0 \\
\theta(0)=(0), \theta(1)=0
\end{gathered}
$$

we obtain this,

$$
\begin{gathered}
\theta^{11}(x)=-\frac{\lambda_{1}(\theta(x))^{n} e^{\frac{\theta(x)}{1+\theta(x)}}}{k\left(1+\lambda_{2} e^{\frac{\theta(x)-1}{1+\epsilon \theta(x)}}\right)}=f(x, \theta) \\
\theta(0)=, \theta^{1}(0)=S_{n}=0
\end{gathered}
$$

Hence we obtain the $2^{\text {nd }}$ initial value problem (IVP) of the form

$$
\begin{aligned}
& Z^{11}=f_{0} Z \\
& Z(0)=0, \quad Z^{1}(0)=I
\end{aligned}
$$

where

$$
f_{\theta}=\frac{-\left(\lambda_{2}\left(1+\in \theta+\epsilon^{2} \theta^{2}\right) e^{\frac{\theta-1}{1+\epsilon \theta}}+1+\epsilon^{2} \theta^{2}+(2 \in+1) \theta\right) \theta^{1} \lambda_{1} e^{\frac{\theta}{e^{1+\epsilon \theta}}}}{k\left(1+\lambda_{2} e^{\frac{\theta-1}{1+\epsilon \theta}}\right)^{2}(1+\in \theta)^{2}}
$$

Reducing Equation (4.1.1) into system of $1^{\text {st }}$ order initial value problem.

Let 


$$
\begin{aligned}
& \theta^{1}=U, \quad \theta(0)=0 \\
& U^{1}=-\frac{\lambda_{1} \theta^{n} e^{\frac{\theta}{1+\epsilon \theta}}}{k\left(1+\lambda_{2} e^{\frac{\theta-1}{1+\epsilon \theta}}\right)}
\end{aligned}
$$

and $2^{\text {nd }}$ initial value problem

Let

$$
\begin{array}{ll}
Z^{1}=p, & Z(0)=0 \\
P^{1}=f_{\theta}(z), & P(0)=1
\end{array}
$$

By Runge-kutta of order four we have

$$
y_{n+1}=y_{n}+\frac{1}{6}\left[k_{1}+2 k_{2}+2 k_{3}+k_{4}\right]
$$

where

$$
\begin{aligned}
& k_{1}=h * f(u[m] \\
& k_{2}=h * f\left(u[m]+\frac{n_{1}}{2}\right) \\
& k_{3}=h * f\left(u[m]+\frac{n_{2}}{2}\right) \\
& k_{4}=h *\left(u[m]+n_{3}\right)
\end{aligned}
$$

Also

$$
n_{1}=h^{*}-\left(\frac{\lambda_{1} \theta_{m}^{n} e^{\frac{\theta_{m}}{1+\varepsilon} \theta_{m}}}{K\left(1+\lambda_{2} e^{\frac{\theta_{m}-1}{1+\varepsilon} \theta_{m}}\right)}\right)
$$




$$
\begin{aligned}
& n_{2}=h^{*}-\left(\frac{\lambda_{1} \theta_{m}^{n}+\frac{\kappa_{1}}{2} e_{1+\varepsilon}^{\frac{\theta_{m}+\frac{\kappa_{1}}{2}}{2+\frac{\kappa_{1}}{2}}}}{K\left(1+\lambda_{2} e_{1+\varepsilon}^{\frac{\theta_{m}+\frac{\kappa_{1}}{2}-1}{\theta_{m}+\frac{\kappa_{1}}{2}}}\right)}\right) \\
& n_{3}=h^{*}-\left(\frac{\lambda_{1} \theta_{m}^{n}+\frac{\kappa_{2}}{2} e_{1+\varepsilon}^{\frac{\theta_{m}+\frac{\kappa_{2}}{2}}{\theta_{m}+\frac{\kappa_{2}}{2}}}}{K\left(1+\lambda_{2} e_{1+\varepsilon}^{\frac{\theta_{m}+\frac{\kappa_{2}}{2}-1}{\theta_{m}+\frac{\kappa_{2}}{2}}}\right)}\right) \\
& n_{4}=h^{*}-\left(\frac{\lambda_{1} \theta_{m}^{n}+\kappa_{3} e^{\frac{\theta_{m}+\kappa_{3}}{1+\varepsilon} \theta_{m}+\kappa_{3}}}{K\left(1+\lambda_{2} e^{\frac{\theta_{m}^{+} \kappa_{3}^{-1}}{1+\varepsilon} \theta_{m}+\kappa_{3}}\right)}\right)
\end{aligned}
$$

A computer program was written to perform the iterative computations.

\section{RESULTS AND DISCUSSION}

The existence and uniqueness of problem is proved by the actual solution. Also the analytical solution of the equation (3.4) was given by Equation (3.1.15) - (3.1.18). For the unsteady state reaction, numerical simulations have been carried out for different values of $\lambda_{1}$, .The numerical evaluations of the unsteady of temperature flame profiles are presented in Figures 1 and 2. It shows that the flames temperature increases with increase in FrankKamenestikii parameter $\lambda_{1}$. For the steady state reaction, numerical calculations have been carried out for different values of $\lambda_{2}$. The numerical results for the steady flames chain reaction concentration profiles are displayed in Figures 3 and 4. It is shown from Figures 3 and 4 that temperature profiles increase with increase in Frank Kamentstkii parameter. 


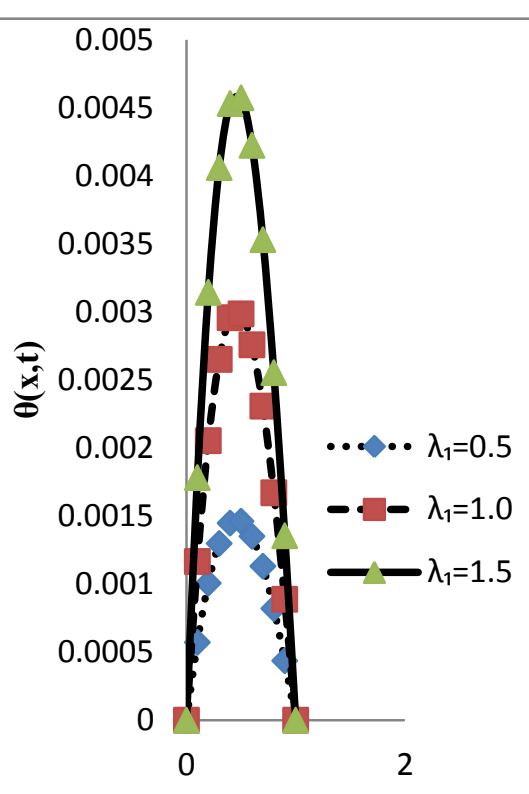

Fig-1: Unsteady temperature profile $\theta(\mathrm{x}, \mathrm{t})$ for equation (3.4) for various values of $\lambda_{1}$.

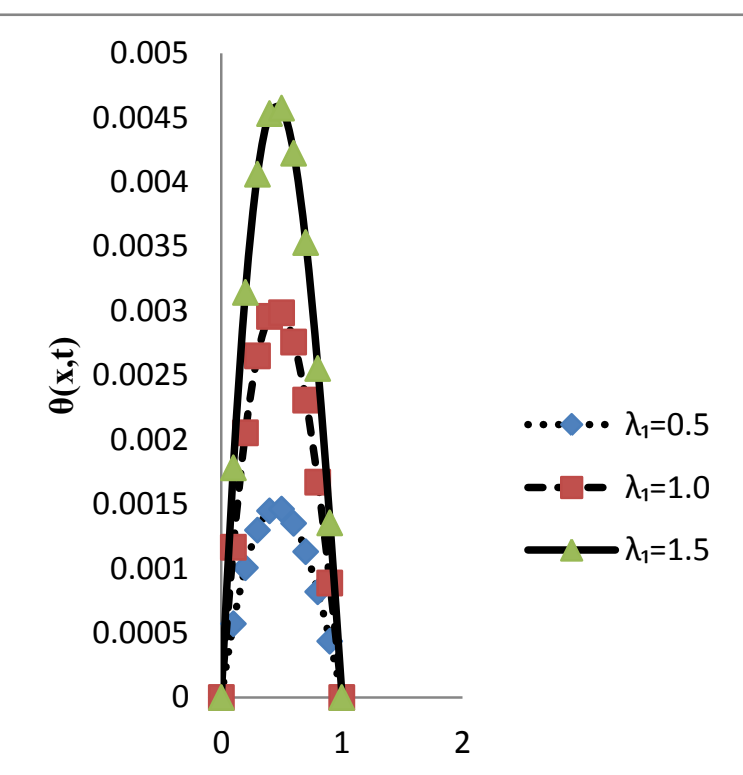

Fig-2: Unsteady temperature profile $\theta(\mathrm{x}, \mathrm{t})$ for equation (3.4) for various values of $\lambda_{1}$. 


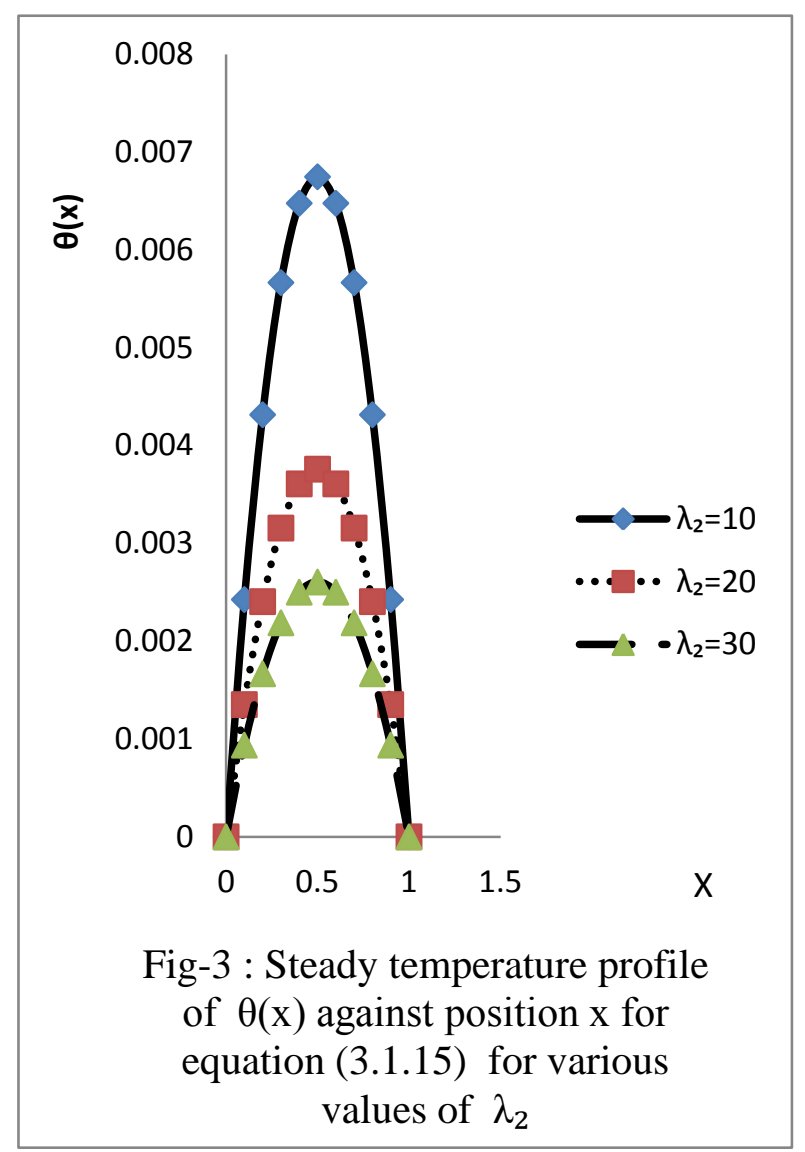

\section{CONCLUSION}

For the burning process associated with chain-breaking and chain-branching kinectics, analytical solution was sought for in steady state. The governing parameter for the problem under study is FrankKamenetskill number. From the studies made on this paper we concluded that Frank-Kamenetskill number enhances the flame temperature.

\section{REFERENCES}

H.B.Kolodner and R.H. Penderson, Point wise bounds for solutions of semi linear parabolic Equations.Journal of Differential Equations, 2(1966),353-364.

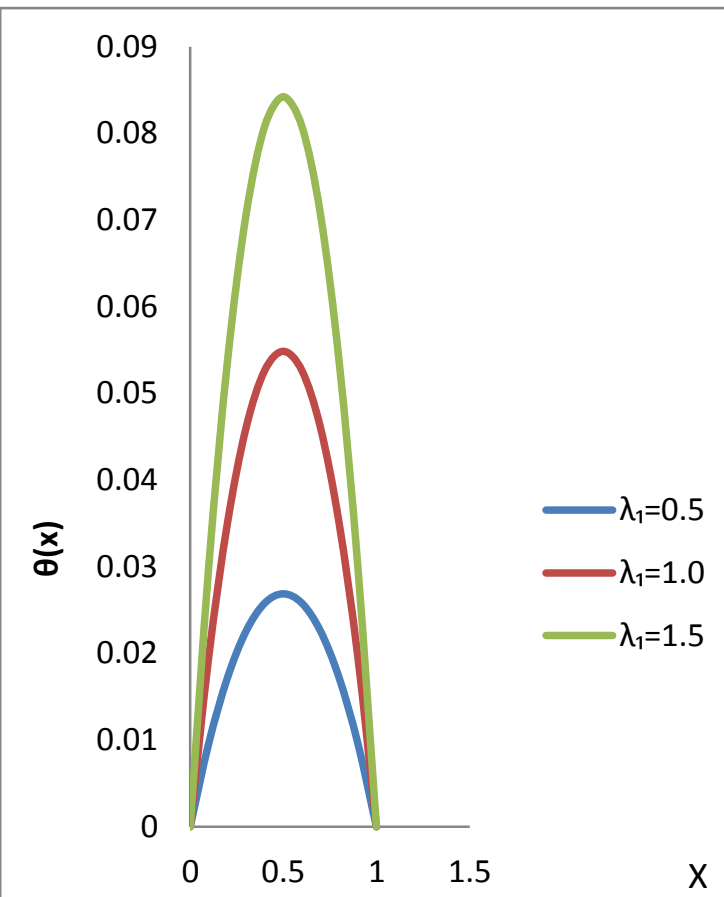

Fig-4: Steady temperature profile of $\theta(\mathrm{x})$ against position $\mathrm{X}$ for equation (3.1.15) for various values of $\lambda_{1}$.
J. D.Buckmaster and G. S. Ludford, Theory of Laminar flames Cambridge university press Cambridge. (1982)

P. O.Olanrewaju, Solution of two step reactions with variable thermal conductivity

Ph.D. Thesis Applied mathematics LAUTECH Ogbomoso Nigeria. (2005).

P. O. Olarenwaju, R. O.,Ayeni, A. O.,Adesanya , O. J Fenugi, and E. A.Adegbile, On the existence and uniqueness of a two-step Arrhenius reaction with strong viscous dissipation term. Research Journal of Applied Sciences, 2(7) (2007), 850-852.

R.O.Ayeni and A. A.Waheed, A Note on Mathematical Model of a Cigarette - like 
Combustion .Appreciating the future of mathematics .Proceedings of the International Conference in Mathematics in honor of Professor E. A. Akinrelere.(2005).

T. Jang and M.I. Raduleson, Dynamics of shock induced ignition in Fickett's model with chain-branching kinetics: Influence of $\chi$. Preprint submitted to the proceeding of combustion institute. (2012)

V. V. Gubernov and J. S. Kim, On the fasttime oscillatory instabilities of Linans diffusion-flame regime. Combust Theory mod. 10 (2006), 747-770.
Z. Huangwei Z. and C.Zheng, Spherical flame initiation and propagation with thermally sensitive intermediate kinetics. Combustion and flame xxx (2011) xxx-Xxx

R.O.,Olayiwola, S.O,Olatunji, S.O.,Ajao, A.A.Waheed and D.L Lanlege, (2013) Effect of Frank-Kamenetskii on the propagation of forward and opposed shouldering combustion Journal of Nigeria Association of Mathematical Physics 23 (2013) 203-214. 